\title{
Minority stress, emotion regulation, and substance misuse in sexual minorities
}

Andrew H. Rogers ${ }^{\mathrm{a}^{*}}$, Ilana Seager ${ }^{\mathrm{a}}$, Nathaniel Haines ${ }^{\mathrm{a}}$, Hunter Hahn ${ }^{\mathrm{a}}$, Amelia Aldao ${ }^{\mathrm{a}}$, Woo-Young Ahn ${ }^{\mathrm{a}^{*}}$

${ }^{a}$ Department of Psychology, The Ohio State University, Columbus, OH 43210

*Corresponding Authors

Andrew H. Rogers, B.A.

Department of Psychology

The Ohio State University

1835 Neil Avenue, Columbus, OH 43210

Email: rogers.1232@,osu.edu, Phone: (614)688-1703

Woo-Young Ahn, Ph.D.

Department of Psychology

The Ohio State University

1835 Neil Avenue, Columbus, OH 43210

Email: ahn.280@,osu.edu, Phone: (614)247-7670

\section{Role of Funding Sources}

The authors disclose no funding sponsor for the design, collection, analysis, interpretation, as well as decision to submit for publication

\section{Contributors}

I.S. and A.A. designed the study and wrote the protocol. A.H.R. conducted literature searches and provided summaries of previous research studies as well as conducted the statistical analysis and wrote the first draft of the manuscript. All authors contributed substantially to and have approved the final manuscript.

\section{Conflicts of Interest}

The authors declare no conflicts of interest.

Word count (Abstract): 241

Word count (Main text): 3500

Number of figures / tables: 4 / 2 


\begin{abstract}
Lesbian, gay, and bisexual (LGB) individuals report higher levels of alcohol and substance misuse than their heterosexual peers, and previous research has linked these increased rates to LGB-specific stressors, termed minority stress. However, not everyone experiencing minority stress reports increased rates of alcohol and substance misuse. Emotion regulation (ER), which plays a critical role in psychopathology generally, is postulated to modulate the link between minority stress and psychopathology. However, it remains largely unknown whether ER plays a role in linking instances of minority stress with substance and alcohol use outcomes. To address the gap, the current study assessed 305 LGB individuals' instances of minority stress, ER, and substance and alcohol use outcomes. We assessed the role of ER in alcohol and substance misuse among LGB individuals using moderated logistic regression and exploratory machine learning analyses. Moderated binary logistic regressions revealed that emotional nonacceptance moderated the relationship between minority stress and problematic substance use, such that those with poor ER were more likely to have problematic substance use. Additionally, deficits in goal-directed behavior moderated the relationship between minority stress and alcohol and substance use, but those with more deficits in goal-directed behavior had better outcomes. Exploratory machine learning analysis revealed multivariate patterns of additional facets of ER and psychiatric symptoms that are predictive of alcohol and substance use. These results highlight the important explanatory role that ER may have between instances of minority stress and substance and alcohol use in LGB individuals.
\end{abstract}

Keywords: Emotion regulation, sexual minorities, alcohol, substance use, machine learning 


\subsection{Introduction}

Lesbian, gay, and bisexual (LGB) individuals report higher rates of psychopathology compared to their heterosexual peers (Coulter et al., 2016; Gilman et al., 2001; Hatzenbuehler et al., 2008; Lee et al., 2016; Meyer, 2003). Researchers have linked these high rates of psychiatric illness to "minority stress," including sexual minority-specific stressors (e.g., discrimination), and coping mechanisms (Meyer, 2003). Alcohol and substance misuse have been specifically linked to these minority stress processes, where higher rates of LGB-specific discrimination contribute to more problematic use (Drazdowski et al., 2016). Additionally, in line with minority stress theory, lower rates of LGB-specific discrimination were associated with decreased rates of problematic alcohol use (Lewis et al., 2016; Meyer, 2003).

Researchers have identified LGB-specific family rejection and victimization as stressors linked to alcohol and substance misuse. Family rejection, defined as a family member's repudiation of an LGB individual due to their sexual orientation (Harter, 1999), is hypothesized to increase internal distress, explaining why some LGB individuals suffer from depression and substance use (Bregman et al., 2013; Ryan et al., 2009; Willoughby et al., 2010). Critically, in a study assessing rates of family rejection in LGB adolescents, $36 \%$ of respondents indicated experiencing at least one negative reaction from a family member following sexual orientation disclosure (Pilkington and D'Augelli, 1995). Additionally, LGB-specific victimization events (e.g. physical violence, verbal harassment) affect up to $80 \%$ of LGB individuals ages 15 21(Pilkington and D'Augelli, 1995), and victimization has been linked to higher rates of mental health problems in general (e.g., depression, post-traumatic stress disorder; Lee et al., 2016), and alcohol and substance use in particular (Willoughby et al., 2010). 
Despite evidence linking LGB-specific instances of discrimination with alcohol and substance misuse, not everyone who experiences discrimination reports increased rates of alcohol and substance use. A promising mechanism for this relationship may be regulatory capacity, as a recent study found that LGB individuals who were better able to adaptively cope with LGB-related discrimination showed better overall mental health outcomes (Kaysen et al., 2014; Nadal et al., 2011). In light of the strong negative emotions associated with LGB discrimination, coping strategies that regulate affect (emotion regulation) may be particularly effective for LGB individuals (Hatzenbuehler et al., 2008).

Emotion regulation (ER), defined as the process by which individuals alter how they experience and express emotions (Gross, 1998), has been implicated in the onset and maintenance of many psychiatric disorders, including alcohol and substance misuse (Aldao et al., 2010). Poor ER has been associated with increased symptoms of anxiety, depression, eating disorders, and alcohol and substance use disorders (Aldao, 2013, 2012; Shadur and Lejuez, 2015). ER is a broad construct encompassing strategies (Aldao et al., 2010), responses to positive affect (Feldman et al., 2008), rumination (Treynor et al., 2003), worry (Meyer et al., 1990), and distress tolerance (Simons and Gaher, 2005). Recent research has identified two specific facets of ER, the ability to tolerate negative emotions as well as distress tolerance (the ability to persist in goal-directed behavior while experiencing negative emotions; Simons and Gaher, 2005), to explain the relationship between stress and alcohol and substance use (Brown et al., 2005; Daughters et al., 2009; Westphal et al., 2017)

A considerable amount of research has linked poor ER to symptoms of psychopathology, yet there is a paucity of work examining these relationships in LGB individuals. Hatzenbuehler and colleagues (2009) found ER to be related to experiences of minority stress, anxiety, and 
depression in LGB individuals as well as finding ER difficulties to significantly mediate the relationship between minority status, anxiety, and depression in LGB adolescents (Hatzenbuehler et al., 2008). Furthermore, previous work has found that LGB individuals often use substances to cope with victimization (Feinstein and Newcomb, 2016), but, to the best of our knowledge, no studies have examined ER to explain why some LGB individuals use substances to cope with minority stress and others do not.

Therefore, to assess the role of ER in alcohol and substance misuse among LGB individuals, the current study examined the moderating role of distress tolerance and the ability to tolerate negative emotions (henceforth referred to as "ER difficulties") to explain the relationship between family rejection, victimization, and substance and/or alcohol misuse in LGB individuals. We hypothesized that for those individuals with ER difficulties, family rejection and victimization would increase substance and/or alcohol misuse compared to those individuals with better ER in these domains.

Additionally, we conducted an exploratory machine learning analysis examining the multivariate patterns of additional facets of ER, as well as psychiatric symptoms, that are most predictive of alcohol and substance use. To the best of our knowledge, this is the first study to use machine learning to examine factors related to alcohol and substance use in an LBG population. Machine learning can identify domains that are most predictive of alcohol and substance use above and beyond those hypothesized. Machine learning is regarded as a promising avenue to identify makers of psychiatric illness, as the analytic methods characterizes multivariate patterns in the data to predict group membership instead of examining each variable individually (Ahn and Vassileva, 2016; Volkow et al., 2015). 


\subsection{Method}

\subsection{Participants}

Participants were drawn from two online studies (both with identical procedures) examining LGB discrimination and sexual orientation self-disclosure. Recruitment occurred through Facebook advertisements, Craigslist posts, and emails to LGB organizations and listservs. Eligible participants needed to: 1) be over the age of 18 years, 2) self-identify as a sexual minority (i.e., lesbian, gay, or bisexual), and 3) currently reside in the United States. Given the reduced control online data collection affords, we took several measures to ensure maximal data integrity. First, if participants did not meet the three criteria above, the survey automatically closed out preventing the collection of any data. We then kept track of IP addresses in order to ensure that people were not answering those questions multiple times until they completed them correctly. Second, we excluded participants who provided incomplete surveys (e.g., did not complete questionnaires), who completed the study multiple times, and who failed more than four "attention questions" designed to catch automated bot programs and disengaged participants (e.g., Hauser and Schwarz, 2016; Oppenheimer et al., 2009). Although gender minority (e.g., transgender, gender non-conforming) participants were included in the larger sample, they were excluded from the present analyses due to sexual orientation-specific stressors likely impacting these participants differently than cisgender participants (Hendricks and Testa, 2012).

The final sample $(N=305)$ had a mean age of 28.68 years $(S D=12.08$ years $)$. Twentythree percent of participants identified as lesbian, $39.3 \%$ as gay, and $37.7 \%$ as bisexual; while $53.3 \%$ identified as female and $46.6 \%$ identified as male. Participants largely identified as Caucasian (83.9\%), non-Hispanic (90.5\%) and were relatively well educated (40.7\% indicated 
having completed at least some college coursework). Moreover, participants resided in a diverse range of locations, with $33.4 \%$ living in a large city, $26.9 \%$ living in a small city, $19.3 \%$ living in a suburban area, $14.8 \%$ living in a small town, and 5.6\% living in a rural area.

\subsection{Procedure}

After providing informed consent, participants completed a battery of self-report questionnaires. All study participation was completed online via Qualtrics, a web-based survey program that meets high standards for data security (SAS 70 Certification; HIPAA-compliant; Huebner et al., 2015). Following study completion, participants were debriefed and given a $\$ 15$ Amazon.com electronic gift card for their participation. This protocol was approved by the Institutional Review Board at the institution where the research was conducted.

\subsection{Self-Report Measures}

Below are the names of the self-report scales used in this study. For more details on the selfreport scales used in this study, please see Supplemental Materials.

2.3.1 Alcohol and substance use. To assess alcohol use, participants completed the Short Michigan Alcohol Screening Test (Selzer et al., 1975), and to assess substance use (all substances other than alcohol and tobacco), participants completed the Drug Abuse Screening Test (Cocco and Carey, 1998).

2.3.2 Minority stress. To assess instances of minority stress, participants completed the Everyday Discrimination Scale (Williams et al., 1997) and the Daily Heterosexist Experiences 
Questionnaire (Balsam et al., 2013), focusing on the family rejection (DHEQ-FAM) and the victimization subscales (DHEQ-VIC).

2.3.3 Emotion regulation. To assess different facets of ER, participants completed the Difficulties with Emotion Regulation Scales (Gratz and Roemer, 2004), focusing Nonacceptance of Emotional Responses (DERS-N) and Difficulties Engaging in Goal Directed Behavior (DERS-G). Both of these subscales have shown considerable overlap with distress tolerance and the ability to tolerate negative emotions (Clen et al., 2011; Daughters et al., 2009). Additionally, participants completed the Emotion Regulation Questionnaire (Gross and John, 2003), the Penn State Worry Questionnaire (Meyer et al., 1990), the Responses to Positive Affect Scale (Feldman et al., 2008), the Ruminative Responses Scale- Brooding (Treynor et al., 2003), and the Brief Resilience Scale (BRS; Smith et al., 2008).

2.3.4 Symptom Measures. To assess psychopathology, participants completed the Center for Epidemiological Studies - Depression Scale (CES-D; Radloff, 1977), the Social Interaction Anxiety Scale (SIAS; Mattick and Clarke, 1998), the Generalized Anxiety Disorder Questionnaire (GAD-7; Spitzer et al., 2006), and the PTSD Checklist - Civilian Version (PCL; Weathers et al., 1991).

\subsection{Data analysis}

Data analysis was conducted using SPSS and R. Moderated binary logistic regression analyses were conducted using the PROCESS macro (Hayes \& Preacher, 2013) to determine the moderating effect of ER between minority stress and problematic alcohol and substance use. 
Group membership based on the moderator was determined using the mean of the moderator $+/$ 1 SD (Hayes \& Preacher, 2013). Due to conceptual overlap between PTSD symptoms and victimization, the moderation analyses were conducted controlling for PCL total score.

To identify ER and minority stress measures which could best predict alcohol and substance use, we took a machine learning approach. We created a separate model for predicting problematic alcohol and substance use, where each model used all ER ( $n=12)$, minority stress $(\mathrm{n}=7)$, and symptom $(\mathrm{n}=4)$ measures to predict problematic alcohol/substance use. Machine learning analyses were conducted using the easyml package (Hendricks \& Ahn, 2017). Specifically, we conducted a penalized (logistic) regression procedure called the least absolute shrinkage and selection operator (LASSO) (Tibshirani, 1996), which is a multiple regression with a penalty term on the sum of absolute values on beta weights, thus making the model more parsimonious and generalizable to new populations. To measure prediction accuracy of the LASSO model, data was split into a training (67\% of data; 204 subjects) and test (33\% of data; 101 subjects) set. We used the Area Under the Curve (AUC) of the Receiver Operating Characteristic (ROC) curve to measure classification accuracy on the test set. One thousand different divisions of training/test sets were tested to check if the performance of LASSO is robust across the divisions. Extensive details on the model fitting procedures are described in a previous study (Ahn et al., 2014) and validated in multiple studies (Ahn et al., 2016; Ahn and Vassileva, 2016; Vilares et al., 2017).

\subsection{Results}

For details on the internal consistency of the self-report scales use, please see Table 1.

\subsection{Correlation coefficients and moderated binary logistic regression results}




\begin{tabular}{lllllllll}
\multicolumn{2}{l}{ Correlations between variables and internal consistency } \\
\hline Measure & 1 & 2 & 3 & 4 & 5 & 6 & 7 & 8 \\
\hline 1. Age & -- & & & & & & & \\
2. DAST & -0.05 & 0.84 & & & & & & \\
3. DERS-G & $-0.22^{* *}$ & 0.07 & 0.89 & & & & & \\
4. DERS-N & $-0.23^{* *}$ & $0.12^{* *}$ & $0.49^{* *}$ & 0.93 & & & & \\
5. Gender & $-0.32^{* *}$ & 0 & $0.21^{* *}$ & $0.15^{* *}$ & -- & & & \\
6. SMAST & 0.06 & $0.26^{* *}$ & 0.10 & $0.14^{* *}$ & $0.12^{* *}$ & 0.84 & & \\
7. DHEQ-VIC & 0.02 & $0.15^{* *}$ & 0.10 & $0.18^{* *}$ & $0.15^{* *}$ & $0.28^{* *}$ & 0.83 & \\
8. DHEQ-FAM & 0.09 & $0.12^{*}$ & -0.03 & $0.16^{* *}$ & -0.08 & $0.16^{* *}$ & $0.38^{* *}$ & 0.79 \\
\hline
\end{tabular}

Table 1. Indicates significance at $* \mathrm{p}<.05 ; * * \mathrm{p}<.01$. Internal consistency (Cronbach's alpha) for the current study is presented on the diagonal when applicable.

Correlations between primary outcome measures and predictors are shown in Table 1.

While some of the measures are moderately correlated (less than 0.4 ), none of the scales are highly correlated (with the exception of the DERS subscales). Binary logistic regressions determined the individual effects of family rejection and victimization on substance and alcohol use. In line with previous research (Bregman et al., 2013; Drazdowski et al., 2016; Ryan et al., 2009; Willoughby et al., 2010), victimization significantly predicted problematic substance use (Odds Ratio [OR]: 1.415, 95\% CI [1.08, 1.85], $p=0.01)$ and alcohol use (OR: 1.799, 95\% CI [1.38-2.30], $p<0.001)$. Additionally, family rejection predicted both problematic substance use $(O R: 1.245,95 \% C I[1.01,1.52], p=0.04)$ and alcohol use $(O R: 1.358,95 \%$ CI [1.08, 1.70], $p=$ 0.008). 


\begin{tabular}{|c|c|c|c|c|}
\hline & & & $95 \%$ Confia & Interval \\
\hline & $B(S E)$ & $p$ value & Lower & Upper \\
\hline Problem Substance & & & & \\
\hline DHEQ-VIC $\times$ DERS-TOT & $-0.008(0.006)$ & $>0.05$ & -0.003 & 0.04 \\
\hline $\begin{array}{l}\text { DHEQ-VIC } \times \text { DERS-G } \\
-1 \text { SD DERS-G }\end{array}$ & $-0.06(0.03)$ & $0.047 *$ & -0.12 & -0.001 \\
\hline DHEQ-VIC & $0.78(0.27)$ & $0.003 *$ & 0.26 & 1.30 \\
\hline Mean DERS-G & & & & \\
\hline DHEQ-VIC & $0.44(0.16)$ & $0.005^{*}$ & 0.14 & 0.75 \\
\hline$+1 S D D E R S-G$ & & & & \\
\hline DHEQ-VIC & $0.11(0.17)$ & $>0.05$ & -0.22 & 0.43 \\
\hline DHEQ-VIC $\times$ DERS- $N$ & $0.03(0.03)$ & $>0.05$ & -0.02 & 0.08 \\
\hline DHEQ-FAM $\times$ DERS-TOT & $0.009(0.005)$ & 0.05 & 0.00 & 0.02 \\
\hline$D H E Q-F A M \times D E R S-G$ & $0.006(0.02)$ & $>0.05$ & -0.04 & 0.05 \\
\hline$D H E Q-F A M \times D E R S-N$ & $0.04(0.02)$ & 0.02 & 0.007 & 0.08 \\
\hline$-1 S D$ DERS-N & & & & \\
\hline DHEQ-FAM & $-0.10(0.16)$ & $>0.05$ & -0.41 & 0.21 \\
\hline Mean DERS-N & & & & \\
\hline $\begin{aligned} & \text { DHEQ-FAM } \\
+ & 1 S D D E R S-N\end{aligned}$ & $0.19(0.12)$ & $>0.05$ & -0.04 & 0.42 \\
\hline DHEQ-FAM & $0.48(0.02)$ & $0.007^{*}$ & 0.13 & 0.83 \\
\hline Problem Alcohol Us & & & & \\
\hline DHEQ-VIC $\times$ DERS-TOT & $-0.01(0.01)$ & 0.08 & -0.02 & 0.001 \\
\hline $\begin{array}{l}\text { DHEQ-VIC } \times \text { DERS-G } \\
-1 \text { SD DERS-G }\end{array}$ & $-0.09(0.03)$ & $0.009^{*}$ & -0.15 & -0.02 \\
\hline DHEQ-VIC & $1.21(0.26)$ & $<0.001^{*}$ & 0.71 & 1.71 \\
\hline Mean DERS-G & & & & \\
\hline $\begin{aligned} & \text { DHEQ-VIC } \\
+ & 1 \text { SD DERS-G }\end{aligned}$ & $0.71(0.15)$ & $<0.001^{*}$ & 0.42 & 1.00 \\
\hline DHEQ-VIC & $0.20(0.19)$ & $>0.05$ & -0.16 & 0.57 \\
\hline DHEQ-VIC $\times$ DERS-N & $-0.01(0.03)$ & $>0.05$ & -0.06 & 0.04 \\
\hline DHEQ-FAM $\times$ DERS-TOT & $0.008(0.005)$ & $>0.05$ & -0.002 & 0.02 \\
\hline$D H E Q-F A M \times D E R S-G$ & $0.008(0.02)$ & $>0.05$ & -0.03 & 0.05 \\
\hline DHEQ-FAM $\times$ DERS-N & $0.02(0.02)$ & $>0.05$ & -0.01 & 0.05 \\
\hline
\end{tabular}

Table 2. Results from moderated binary logistic regressions, with ER variables (goal-directed behavior (DERS-G), emotional non-acceptance (DERS-N)) as moderators between family rejection (DHEQ-FAM)/victimization (DHEQ-VIC) and alcohol/substance use. * indicate a significant result $(p<0.05)$

To investigate whether problematic substance and alcohol use was influenced by ER deficits, we used moderated binary logistic regressions (see Table 2 for full moderation results). A significant family rejection by emotional non-acceptance interaction significantly predicted substance use problems $(p=0.02)$. As expected, for those with high emotional non-acceptance (1 SD above the mean), family rejection was a significant predictor of problematic substance use 
$(B=0.48,95 \% C I[0.13,0.83], p=0.007)$, but this relationship was non-significant for those with average and 1SD below emotional non-acceptance $(p>0.05$, see Figure 1). Emotional nonacceptance did not significantly moderate the relationship between family rejection and problematic alcohol use $(p>0.05)$. Additionally, there were no significant interactions between family rejection and deficits in goal-directed behavior predicting problematic alcohol nor substance use ( $p s>0.05)$.

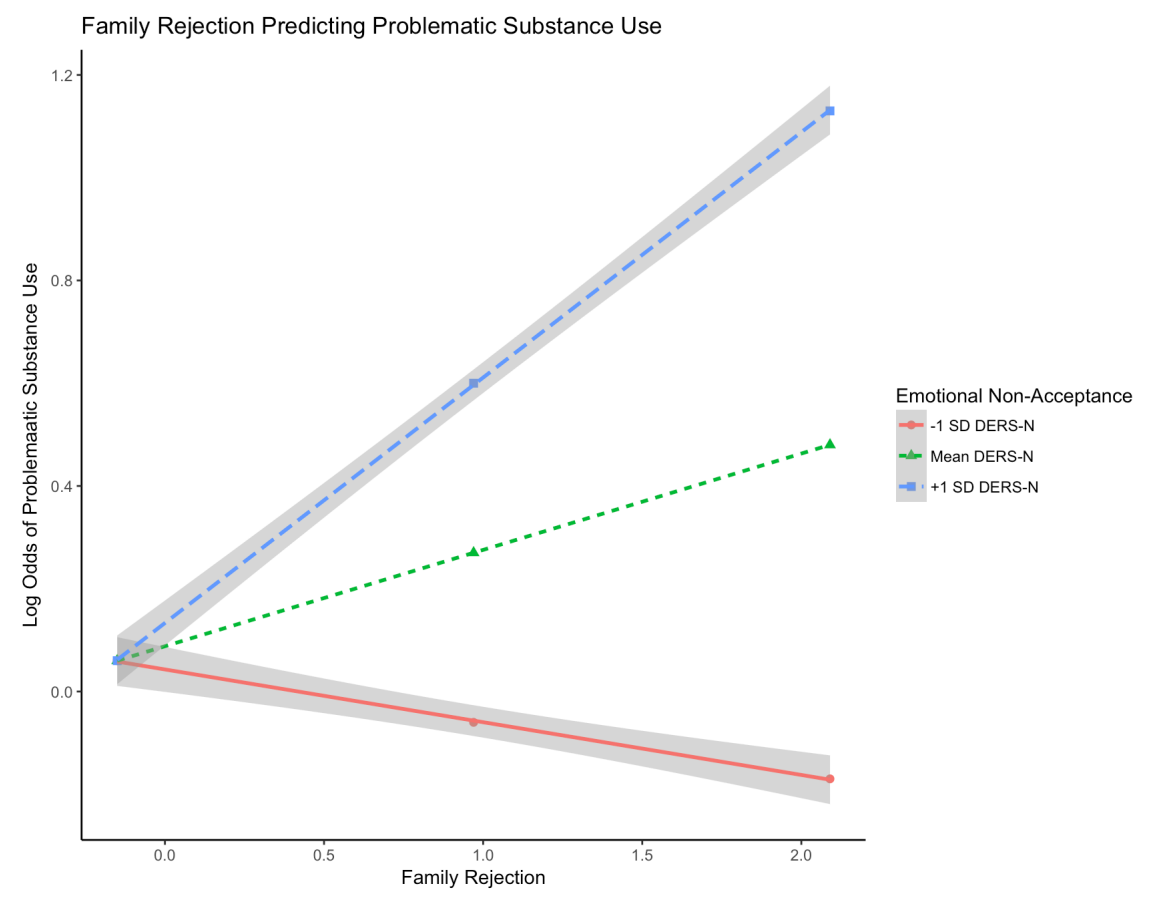

Figure 1. Significant interaction of family rejection and emotional non-acceptance predicting log odds of problematic substance use. The green line with triangles represents those at the mean emotional non-acceptance, the red line with squares represents those participants 1 standard deviation (SD) below the mean on emotional non-acceptance, and the blue line with squares represents those participants $1 \mathrm{SD}$ above the mean. Higher scores indicate more ER difficulties. Lines represent best-fitted regression lines ( $\pm 95 \%$ confidence interval) for each of the groups.

A significant victimization by deficits in goal-directed behavior interaction predicted problematic substance use $(\mathrm{p}=0.047$; see Figure $2 \mathrm{a})$. Contrary to the proposed hypothesis, victimization was a significant predictor of problematic substance use for those with low (-1SD) 
deficits in goal-directed behavior scores $(B=0.78,95 \% C I[0.26,1.30], p=0.003)$ and average deficits in goal-directed behavior scores $(B=0.44,95 \% C I[0.14,0.75], p=0.004)$, but not for those with high $(+1 \mathrm{SD})$ deficits in goal-directed behavior scores $(p>0.05)$. A significant victimization by deficits in goal-directed behavior interaction also predicted problematic alcohol use ( $p=0.009$; see Figure $2 b)$. Similar to the findings for substance use and contrary to the initial hypotheses, victimization predicted problematic alcohol use for those with low (-1SD) DERS goal-directed behavior scores $(B=1.21,95 \% C I[0.71,1.71], p<0.001)$, and average DERS goaldirected behavior scores $(B=0.71,95 \% C I[0.42,1.00], p<0.001)$, but not for those with high $(+1 \mathrm{SD})$ DERS goal-directed behavior scores $(p>0.05)$. There were no significant interactions between victimization and DERS emotional non-acceptance predicting problematic alcohol use nor substance use $(p s>0.05)$.
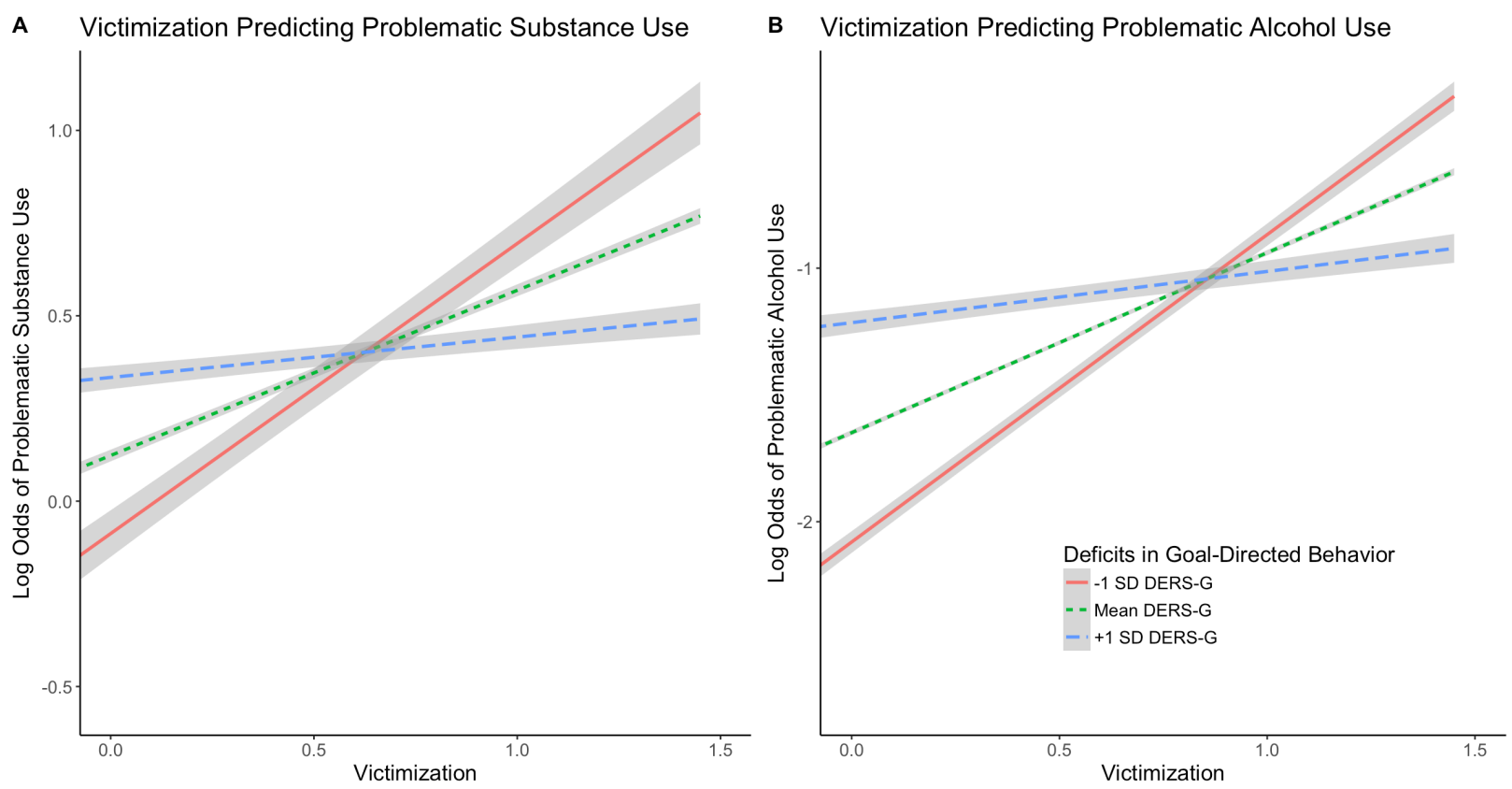

Figure 2. a) Significant interaction of victimization and deficits in goal-directed behavior predicting odds of problematic substance use. Lines represent best-fitted regression lines $( \pm 95 \%$ confidence interval) for each of the three groups. b) Significant interaction of victimization and deficits in goal-directed behavior predicting odds of problematic alcohol use. Lines represent best-fitted regression lines ( $\pm 195 \%$ confidence interval) for each of the three groups. 


\subsection{Machine learning results}

Figure 3 shows the performance of machine learning, predicting problematic alcohol use (Figure 3A and 3B; mean AUCs of 0.76 and 0.66 in training and test sets, respectively) and problematic substance use (Figure 3C and 3D; mean AUCs of 0.69 and 0.60 in training and test sets, respectively) with greater than chance accuracy $(\mathrm{AUC}=0.5)$. Figure 4 shows the multivariate patterns of self-report measures that were most predictive of alcohol use (Figure 4A) and substance use (Figure 4B). EDS total score, DHEQ discrimination, and PCL total score positively predicted problematic substance use. On the other hand, RPA emotional dampening, EDS total, DERS awareness, PCL total, and ERQ suppression positively predicted problematic alcohol use, while DHEQ vicarious trauma individually negatively predicted problematic alcohol use.

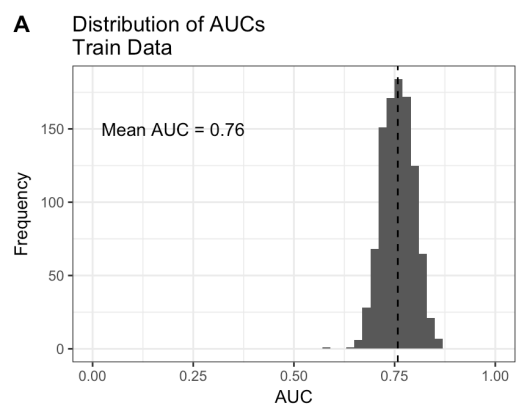

B Distribution of AUCs
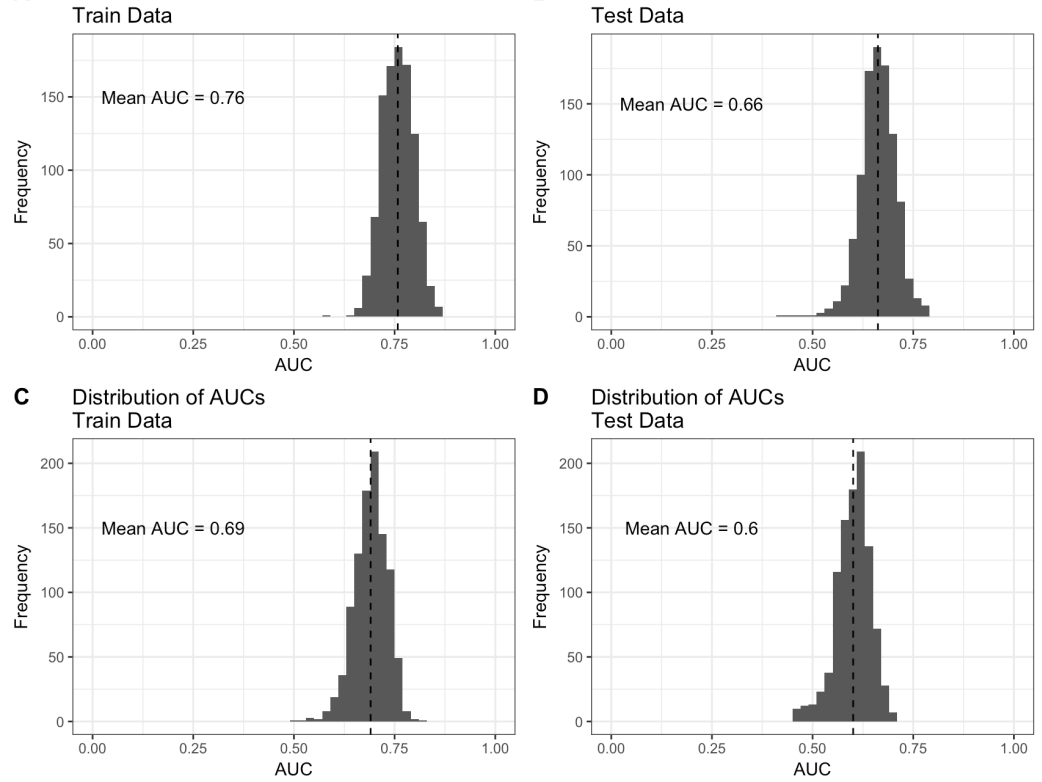

D Distribution of AUCs

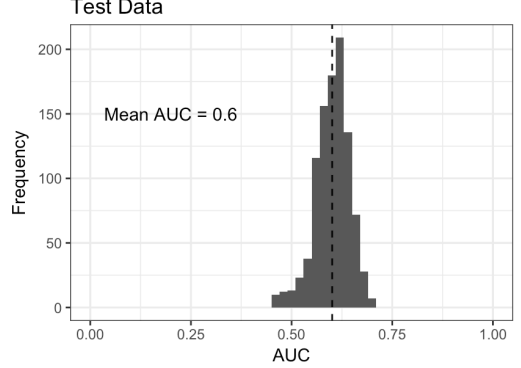

Figure 3. Problematic (A and B) alcohol and (C and D) substance use classifier prediction accuracy across 1,000 permutations of the training/test sets. Distributions show the variance in the area under the curve of the receiver operating characteristic (AUC) estimate across all splits, and dotted lines represent the mean performance. An AUC of 0.5 is expected by a completely random model, while an AUC of 1 indicates perfect prediction accuracy. 
A

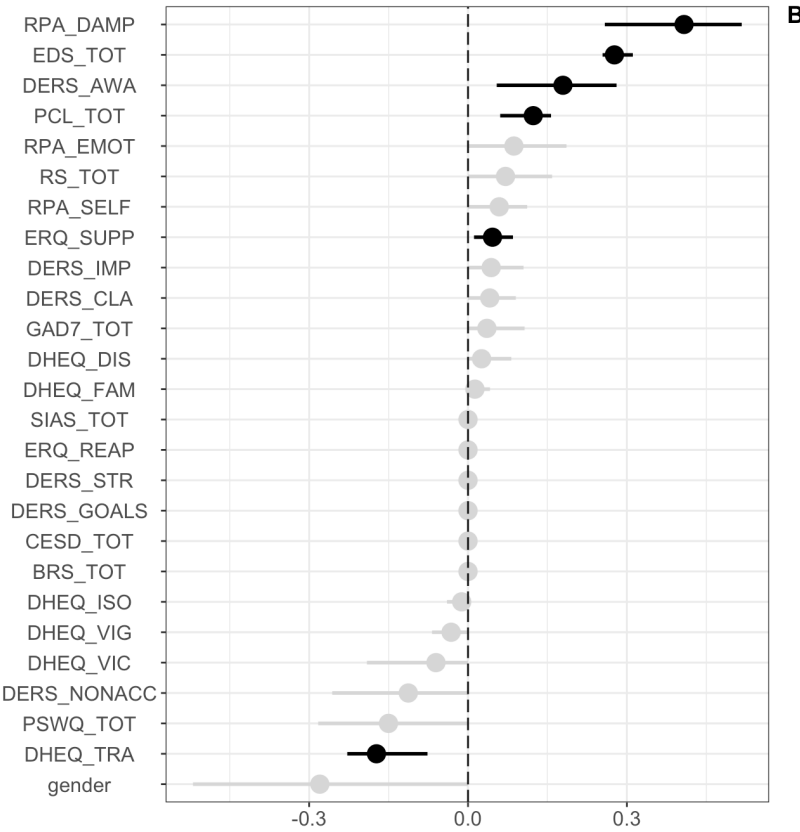

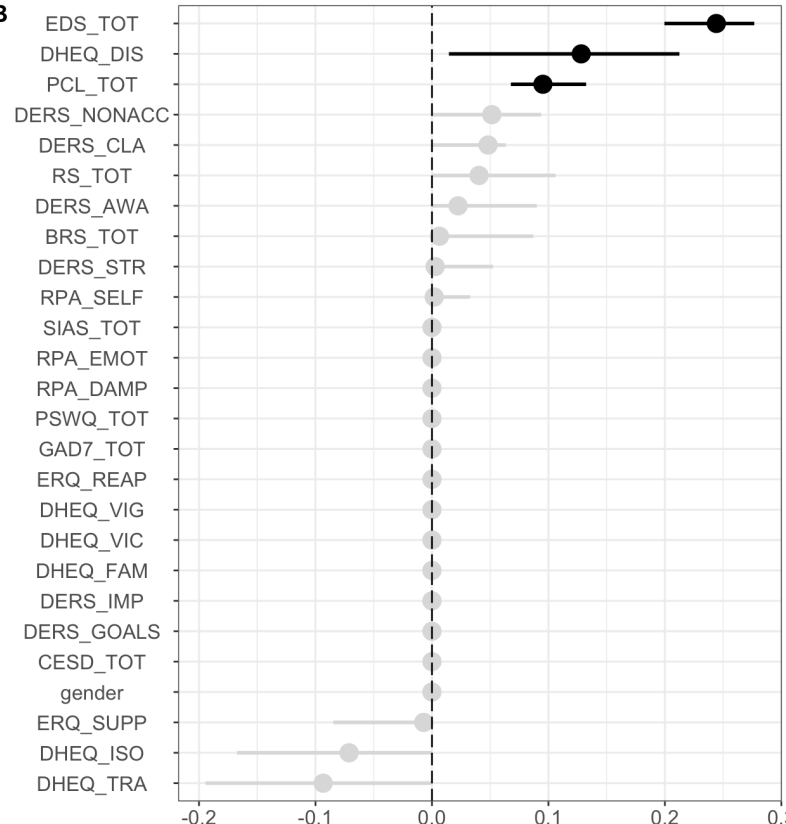

Figure 4. Beta weights for (A) problematic alcohol and (B) substance use classifiers. Darkshaded beta weights survived the LASSO penalty (indicating statistically significant), and lightshaded beta weights were shrunk to 0 . Error bars represent $95 \%$ confidence intervals. Dampening response to positive affect (RPA-DAMP), discrimination (EDS-TOT), emotional awareness (DERS-AWA), PTSD symptoms (PCL-TOT) and habitual use of suppression (ERQSUPP) positively predicted alcohol use and vicarious trauma (DHEQ-TRA) negatively predicted alcohol use. Discrimination (EDS-TOT, DHEQ-DIS) and PTSD symptoms also positively predicted substance use.

\subsection{Discussion}

The goal of the present study was to test whether ER difficulties significantly moderated the relationship between minority status and problematic alcohol and substance use in LGB individuals using moderated binary logistic regressions and machine learning analyses. In line with previous research suggesting instances of LGB-specific discrimination are associated with increased alcohol and substance use (Bregman et al., 2013; Ryan et al., 2009; Willoughby et al., 2010), this investigation also found additional support for this link between LGB-related discrimination, and alcohol- and substance-use. 
Additionally, the present study provided evidence to support ER as an important mechanism explaining alcohol and substance use in response to minority stress. As expected, in the context of family rejection, those with high levels of emotional non-acceptance were more likely to have problematic substance use compared to those with low levels of emotional nonacceptance. This relationship was not significant for problematic alcohol use, which could stem from a number of different factors that affect alcohol use in LGB individuals. For example, due to a long history of discrimination of LGBT people who openly shared their sexual orientation, bars, clubs, and speakeasies have long served as a central hub for LGBT social life (Chauncey, 1994). Thus, drinking alcohol has become a normal part of socializing with other members of the LGB community (Coulter et al., 2016), so it is possible that they did not view their drinking as problematic. Further, although family rejection was a significant individual predictor of problematic alcohol use, emotional non-acceptance did not serve as a moderator, suggesting that the mechanism linking family rejection to alcohol use may not involve emotional nonacceptance.

Emotional non-acceptance also did not significantly moderate the relationship between victimization and substance use, as well as the relationship between victimization and alcohol use. It is possible that the link between victimization and both substance and alcohol use is not related to emotional non-acceptance. Increases in negative affect are largely associated with poor ER (Aldao and Nolen-Hoeksema, 2010), and victimization, as a source of increased negative affect, is present for a significant number of LGB individuals (Pilkington and D'Augelli, 1995). It is therefore possible that individuals may choose to use substances to decrease their negative affect (Feinstein and Newcomb, 2016), regardless of their ability to tolerate negative emotions. ER deficits have been shown to both increase vulnerability for using substances and alcohol to 
cope as well as developing a substance and/or alcohol use disorder (Shadur and Lejuez, 2015); more research in this area should elucidate the temporal order of discrimination and ER, as well as the function of alcohol and substance use in the context of minority stress.

Deficits in goal-directed behavior showed consistent associations for victimization predicting both alcohol and substance use. Contrary to hypotheses, in both instances those with better goal-directed behavior were more likely to have problematic substance and alcohol use than those with poorer goal-directed behavior. However, while associations between goaldirected behavior deficits, alcohol, and substance use have been positively associated (Daughters et al., 2009), there is mixed evidence as to the function of goal-directed behavior in problematic use. One explanation for the discrepant results comes from the Theory of Planned Behavior, which asserts that better goal directed behavior may be important in onset of problematic drinking, as people have to distinguish between desire to use, intention to use, and consideration of social influences (Esposito et al., 2016; Gabbiadini et al., 2017). Additionally, people who use alcohol and substances often over-value the rewards from use, which may actually enhance goaldirected behavior when seeking out alcohol and substances (Murphy et al., 2012). Therefore, based on the results from this study, it is critical for future studies to further assess goal-directed behavior to determine whether problematic use may be primarily driven by goal-directed behavior or habit.

Finally, results from the machine learning analyses identified multivariate patterns of predictors of both problematic alcohol and substance use not tested in the original hypothesis. In line with much of the extant literature (Bregman et al., 2013; Ryan et al., 2009; Willoughby et al., 2010), LGB-specific discrimination as well as PTSD symptoms positively predicted both alcohol and substance misuse, suggesting that these may be common risk factors for both 
categories of use. However, while some of the associations are consistent between alcohol and substance misuse, generally these results show that substance use may be more related to poor ER generally, which is consistent with past research (Weiss et al., 2015a, 2015b). Future research should further examine these constructs and how they contribute to the development and maintenance of substance and alcohol problems in LGB individuals using experimental, longitudinal, and ecological momentary assessment methods.

\subsection{Strengths, Limitations, and Future Directions}

This study benefits from a number of strengths, including the large sample size as well as providing initial evidence for ER as a potential mechanism explaining links between LGBspecific discrimination and alcohol and substance misuse. However, there are limitations that must be noted. First, the data collected is cross-sectional, prohibiting causal and temporal claims to be made regarding the relationships. Additionally, since the study did not explicitly recruit LGB substance and alcohol users, there was limited variability in the scales assessing alcohol and substance misuse. Finally, although numerous steps were conducted to minimize lack of experimental control for an online study, it is still possible that participants answered the questionnaires without spending the time to understand the question asked.

Future studies should seek to further elucidate the role that ER plays to explain why LGB-specific discrimination events lead to elevated rates of alcohol and substance misuse. Both laboratory experimental studies as well as longitudinal studies could be used to establish both temporal order of ER, minority stress, alcohol, and substance use, as well as determine causation in these relationships. Future research should also more closely examine specific ER strategies that serve as either risk or protective factors for alcohol and substance misuse. Findings from 
these studies can improve current psychiatric treatments for alcohol and substance misuse, as well as provide culturally competent guidance for therapists working with LGB clients living with alcohol and substance use disorders.

\subsection{Conclusion}

To our knowledge, this is the first study to examine ER as a moderator between experiences of minority stress and problematic alcohol and substance use in LGB individuals.

Results from the study provide initial evidence to support ER as an important mechanism between minority stress, alcohol, and substance use. Assessing substance use and ER in a clinical context with LGB individuals may be important to achieve the best possible clinical outcomes. 


\section{References}

Ahn, W.-Y., Ramesh, D., Moeller, F.G., Vassileva, J., 2016. Utility of Machine-Learning Approaches to Identify Behavioral Markers for Substance Use Disorders: Impulsivity Dimensions as Predictors of Current Cocaine Dependence. Front. Psychiatry 7, 34. doi:10.3389/fpsyt.2016.00034

Ahn, W.-Y., Vassileva, J., 2016. Machine-learning identifies substance-specific behavioral markers for opiate and stimulant dependence. Drug Alcohol Depend. 161, 247-257. doi:10.1016/j.drugalcdep.2016.02.008

Aldao, A., 2013. The Future of Emotion Regulation Research: Capturing Context. Perspect. Psychol. Sci. 8, 155-172. doi:10.1177/1745691612459518

Aldao, A., 2012. Emotion Regulation Strategies as Transdiagnostic Processes: A Closer Look at the Invariance of Their Form and Function. Span. J. Clin. Psychol. 17, 261-277.

Aldao, A., Nolen-Hoeksema, S., 2010. Specificity of cognitive emotion regulation strategies: a transdiagnostic examination. Behav. Res. Ther. 48, 974-983. doi:10.1016/j.brat.2010.06.002

Aldao, A., Nolen-Hoeksema, S., Schweizer, S., 2010. Emotion-regulation strategies across psychopathology: A meta-analytic review. Clin. Psychol. Rev. 30, 217-237. doi:10.1016/j.cpr.2009.11.004

Balsam, K.F., Beadnell, B., Molina, Y., 2013. The Daily Heterosexist Experiences Questionnaire: Measuring Minority Stress Among Lesbian, Gay, Bisexual, and Transgender Adults. Meas. Eval. Couns. Dev. Off. Publ. Assoc. Meas. Eval. Couns. Dev. 46, 3-25. doi:10.1177/0748175612449743

Bregman, H.R., Malik, N.M., Page, M.J.L., Makynen, E., Lindahl, K.M., 2013. Identity profiles in lesbian, gay, and bisexual youth: the role of family influences. J. Youth Adolesc. 42, 417-430. doi:10.1007/s10964-012-9798-z

Brown, R.A., Lejuez, C.W., Kahler, C.W., Strong, D.R., Zvolensky, M.J., 2005. Distress tolerance and early smoking lapse. Clin. Psychol. Rev. 25, 713-733. doi:10.1016/j.cpr.2005.05.003

Chauncey, G., 1994. Gay New York: Gender, Urban Culture, and the Making of the Gay Male World, 1890-1940. Basic Books.

Clen, S.L., Mennin, D.S., Fresco, D.M., 2011. Major Depressive Disorder, in: Distress Tolerance: Theory, Research, and Clinical Applications. The Guilford Press.

Cocco, K.M., Carey, K.B., 1998. Psychometric properties of the Drug Abuse Screening Test in psychiatric outpatients. Psychol. Assess. 10, 408-414. doi:10.1037/1040-3590.10.4.408

Coulter, R.W.S., Marzell, M., Saltz, R., Stall, R., Mair, C., 2016. Sexual-orientation differences in drinking patterns and use of drinking contexts among college students. Drug Alcohol Depend. 160, 197-204. doi:10.1016/j.drugalcdep.2016.01.006

Daughters, S.B., Reynolds, E.K., MacPherson, L., Kahler, C.W., Danielson, C.K., Zvolensky, M., Lejuez, C.W., 2009. Distress tolerance and early adolescent externalizing and internalizing symptoms: The moderating role of gender and ethnicity. Behav. Res. Ther. 47, 198-205. doi:10.1016/j.brat.2008.12.001

Drazdowski, T.K., Perrin, P.B., Trujillo, M., Sutter, M., Benotsch, E.G., Snipes, D.J., 2016. Structural equation modeling of the effects of racism, LGBTQ discrimination, and internalized oppression on illicit drug use in LGBTQ people of color. Drug Alcohol Depend. 159, 255-262. doi:10.1016/j.drugalcdep.2015.12.029 
Esposito, G., van Bavel, R., Baranowski, T., Duch-Brown, N., 2016. Applying the Model of Goal-Directed Behavior, Including Descriptive Norms, to Physical Activity Intentions: A Contribution to Improving the Theory of Planned Behavior. Psychol. Rep. 119, 5-26. doi:10.1177/0033294116649576

Feinstein, B.A., Newcomb, M.E., 2016. The role of substance use motives in the associations between minority stressors and substance use problems among young men who have sex with men. Psychol. Sex. Orientat. Gend. Divers. 3, 357-366. doi:10.1037/sgd0000185

Feldman, G.C., Joormann, J., Johnson, S.L., 2008. Responses to Positive Affect: A Self-Report Measure of Rumination and Dampening. Cogn. Ther. Res. 32, 507-525. doi:10.1007/s10608-006-9083-0

Gabbiadini, A., Cristini, F., Scacchi, L., Monaci, M.G., 2017. Testing the Model of GoalDirected Behavior for Predicting Binge Drinking Among Young People. Subst. Use Misuse 52, 493-506. doi:10.1080/10826084.2016.1245335

Gilman, S.E., Cochran, S.D., Mays, V.M., Hughes, M., Ostrow, D., Kessler, R.C., 2001. Risk of psychiatric disorders among individuals reporting same-sex sexual partners in the National Comorbidity Survey. Am. J. Public Health 91, 933-939.

Gratz, K.L., Roemer, L., 2004. Multidimensional Assessment of Emotion Regulation and Dysregulation: Development, Factor Structure, and Initial Validation of the Difficulties in Emotion Regulation Scale. J. Psychopathol. Behav. Assess. 26, 41-54. doi:10.1023/B:JOBA.0000007455.08539.94

Gross, J.J., 1998. The emerging field of emotion regulation: An integrative review. Rev. Gen. Psychol. 2, 271-299. doi:10.1037/1089-2680.2.3.271

Gross, J.J., John, O.P., 2003. Individual differences in two emotion regulation processes: Implications for affect, relationships, and well-being. J. Pers. Soc. Psychol. 85, 348-362. doi:10.1037/0022-3514.85.2.348

Harter, S., 1999. The Construction of the Self: A Developmental Perspective. Guilford Press. Hatzenbuehler, M.L., Dovidio, J.F., Nolen-Hoeksema, S., Phills, C.E., 2009. An Implicit Measure of Anti-Gay Attitudes: Prospective Associations with Emotion Regulation Strategies and Psychological Distress. J. Exp. Soc. Psychol. 45, 1316-1320. doi:10.1016/j.jesp.2009.08.005

Hatzenbuehler, M.L., McLaughlin, K.A., Nolen-Hoeksema, S., 2008. Emotion regulation and internalizing symptoms in a longitudinal study of sexual minority and heterosexual adolescents. J. Child Psychol. Psychiatry 49, 1270-1278. doi:10.1111/j.14697610.2008.01924.x

Hauser, D.J., Schwarz, N., 2016. Attentive Turkers: MTurk participants perform better on online attention checks than do subject pool participants. Behav. Res. Methods 48, 400-407. doi:10.3758/s13428-015-0578-z

Hendricks, M.L., Testa, R.J., 2012. A conceptual framework for clinical work with transgender and gender nonconforming clients: An adaptation of the Minority Stress Model. Prof. Psychol. Res. Pract. 43, 460-467. doi:10.1037/a0029597

Hendricks, P., Ahn, W.-Y., 2017. Easyml: Easily Build And Evaluate Machine Learning Models. bioRxiv 137240. doi:10.1101/137240

Huebner, D.M., Thoma, B.C., Neilands, T.B., 2015. School victimization and substance use among lesbian, gay, bisexual, and transgender adolescents. Prev. Sci. Off. J. Soc. Prev. Res. 16, 734-743. doi:10.1007/s11121-014-0507-x 
Kaysen, D.L., Kulesza, M., Balsam, K.F., Rhew, I.C., Blayney, J.A., Lehavot, K., Hughes, T.L., 2014. Coping as a mediator of internalized homophobia and psychological distress among young adult sexual minority women. Psychol. Sex. Orientat. Gend. Divers. 1, 225-233. doi:10.1037/sgd0000045

Lee, J.H., Gamarel, K.E., Bryant, K.J., Zaller, N.D., Operario, D., 2016. Discrimination, Mental Health, and Substance Use Disorders Among Sexual Minority Populations. LGBT Health 3, 258-265. doi:10.1089/lgbt.2015.0135

Lewis, R.J., Mason, T.B., Winstead, B.A., Gaskins, M., Irons, L.B., 2016. Pathways to Hazardous Drinking Among Racially and Socioeconomically Diverse Lesbian Women: Sexual Minority Stress, Rumination, Social Isolation, and Drinking to Cope. Psychol. Women Q. 40, 564-581. doi:10.1177/0361684316662603

Mattick, R.P., Clarke, J.C., 1998. Development and validation of measures of social phobia scrutiny fear and social interaction anxiety1. Behav. Res. Ther. 36, 455-470. doi:10.1016/S0005-7967(97)10031-6

Meyer, I.H., 2003. Prejudice, Social Stress, and Mental Health in Lesbian, Gay, and Bisexual Populations: Conceptual Issues and Research Evidence. Psychol. Bull. 129, 674-697. doi:10.1037/0033-2909.129.5.674

Meyer, T.J., Miller, M.L., Metzger, R.L., Borkovec, T.D., 1990. Development and validation of the penn state worry questionnaire. Behav. Res. Ther. 28, 487-495. doi:10.1016/00057967(90)90135-6

Murphy, A., Taylor, E., Elliott, R., 2012. The detrimental effects of emotional process dysregulation on decision-making in substance dependence. Front. Integr. Neurosci. 6. doi:10.3389/fnint.2012.00101

Nadal, K.L., Wong, Y., Issa, M.-A., Meterko, V., Leon, J., Wideman, M., 2011. Sexual Orientation Microaggressions: Processes and Coping Mechanisms for Lesbian, Gay, and Bisexual Individuals. J. LGBT Issues Couns. 5, 21-46. doi:10.1080/15538605.2011.554606

Oppenheimer, D.M., Meyvis, T., Davidenko, N., 2009. Instructional manipulation checks: Detecting satisficing to increase statistical power. J. Exp. Soc. Psychol. 45, 867-872.

Pilkington, N.W., D’Augelli, A.R., 1995. Victimization of lesbian, gay, and bisexual youth in community settings. J. Community Psychol. 23, 34-56. doi:10.1002/15206629(199501)23:1<34::AID-JCOP2290230105>3.0.CO;2-N

Radloff, L.S., 1977. The CES-D Scale: A Self-Report Depression Scale for Research in the General Population. Appl. Psychol. Meas. 1, 385-401. doi:10.1177/014662167700100306

Ryan, C., Huebner, D., Diaz, R.M., Sanchez, J., 2009. Family Rejection as a Predictor of Negative Health Outcomes in White and Latino Lesbian, Gay, and Bisexual Young Adults. Pediatrics 123, 346-352. doi:10.1542/peds.2007-3524

Selzer, M.L., Vinokur, A., van Rooijen, L., 1975. A self-administered Short Michigan Alcoholism Screening Test (SMAST). J. Stud. Alcohol 36, 117-126.

Shadur, J.M., Lejuez, C.W., 2015. Adolescent Substance Use and Comorbid Psychopathology: Emotion Regulation Deficits as a Transdiagnostic Risk Factor. Curr. Addict. Rep. 2, 354363. doi:10.1007/s40429-015-0070-y

Simons, J.S., Gaher, R.M., 2005. The Distress Tolerance Scale: Development and Validation of a Self-Report Measure. Motiv. Emot. 29, 83-102. doi:10.1007/s11031-005-7955-3 
Smith, B.W., Dalen, J., Wiggins, K., Tooley, E., Christopher, P., Bernard, J., 2008. The brief resilience scale: Assessing the ability to bounce back. Int. J. Behav. Med. 15, 194-200. doi:10.1080/10705500802222972

Spitzer, R.L., Kroenke, K., Williams, J.B.W., Löwe, B., 2006. A Brief Measure for Assessing Generalized Anxiety Disorder: The GAD-7. Arch. Intern. Med. 166, 1092-1097. doi:10.1001/archinte.166.10.1092

Treynor, W., Gonzalez, R., Nolen-Hoeksema, S., 2003. Rumination Reconsidered: A Psychometric Analysis. Cogn. Ther. Res. 27, 247-259. doi:10.1023/A:1023910315561

Vilares, I., Wesley, M.J., Ahn, W.-Y., Bonnie, R.J., Hoffman, M., Jones, O.D., Morse, S.J., Yaffe, G., Lohrenz, T., Montague, P.R., 2017. Predicting the knowledge-recklessness distinction in the human brain. Proc. Natl. Acad. Sci. 114, 3222-3227. doi:10.1073/pnas.1619385114

Volkow, N.D., Koob, G., Baler, R., 2015. Biomarkers in Substance Use Disorders. ACS Chem. Neurosci. 6, 522-525. doi:10.1021/acschemneuro.5b00067

Weathers, F.W., Huska, J.A., Keane, T.M., 1991. PCL-C for DSM-IV. Boston Natl. Cent. PTSD-Behav. Sci. Div.

Weiss, N.H., Sullivan, T.P., Tull, M.T., 2015a. Explicating the role of emotion dysregulation in risky behaviors: A review and synthesis of the literature with directions for future research and clinical practice. Curr. Opin. Psychol. 3, 22-29. doi:10.1016/j.copsyc.2015.01.013

Weiss, N.H., Williams, D.C., Connolly, K.M., 2015b. A Preliminary Examination of Negative Affect, Emotion Dysregulation, and Risky Behaviors Among Military Veterans in Residential Substance Abuse Treatment. Mil. Behav. Health 3, 212-218. doi:10.1080/21635781.2015.1038405

Westphal, M., Aldao, A., Jackson, C., 2017. Emotion dysregulation in comorbid posttraumatic stress disorder and substance use disorders: A narrative review. Mil. Psychol. 29, 216233. doi: $10.1037 / \mathrm{mil} 0000157$

Williams, D.R., Yan Yu, null, Jackson, J.S., Anderson, N.B., 1997. Racial Differences in Physical and Mental Health: Socio-economic Status, Stress and Discrimination. J. Health Psychol. 2, 335-351. doi:10.1177/135910539700200305

Willoughby, B.L.B., Doty, N.D., Malik, N.M., 2010. Victimization, Family Rejection, and Outcomes of Gay, Lesbian, and Bisexual Young People: The Role of Negative GLB Identity. J. GLBT Fam. Stud. 6, 403-424. doi:10.1080/1550428X.2010.511085 